\title{
Hybrid Ring- and Tree-Topology RoF Transmission System with Disconnection Protection
}

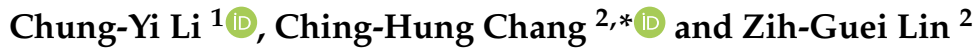 \\ 1 Department of Communication Engineering, National Taipei University, New Taipei City 23741, Taiwan; \\ cyli@gm.ntpu.edu.tw \\ 2 Department of Electrical Engineering, National Chiayi University, Chiayi 60004, Taiwan; \\ tklingary@gmail.com \\ * Correspondence: chchang@mail.ncyu.edu.tw
}

check for updates

Citation: Li, C.-Y.; Chang, C.-H.; Lin, Z.-G. Hybrid Ring- and TreeTopology RoF Transmission System with Disconnection Protection. Photonics 2021, 8, 515. https:// doi.org/10.3390/photonics 8110515

Received: 31 August 2021

Accepted: 10 November 2021

Published: 16 November 2021

Publisher's Note: MDPI stays neutral with regard to jurisdictional claims in published maps and institutional affiliations.

Copyright: (c) 2021 by the authors. Licensee MDPI, Basel, Switzerland. This article is an open access article distributed under the terms and conditions of the Creative Commons Attribution (CC BY) license (https:// creativecommons.org/licenses/by/ $4.0 /)$.

\begin{abstract}
This paper proposes a hybrid ring- and tree-topology radio over fiber (RoF) transmission system with self-disconnection protection that can support the high distribution density of base stations (BSs) in a metropolitan area and strengthen the network quality of service through selfdisconnection protection. The number of supportable BS in the system can be increased significantly by integrating the time- and wavelength-division multiplexing techniques and properly utilizing a new-generation single-line bidirectional add/drop multiplexer (SBOADM) into the proposed system. Moreover, when the ring-fiber link of the system is interrupted for any reason, the system operator can recover the broken connections quickly only by transforming an optical switch state at the CO end to allow the downlink optical signals to transmit along the clockwise and counterclockwise directions of the ring-fiber link simultaneously. In this case, the downstream optical signals can be delivered to each set of BS-groups through the two-way transmission characteristics of the SBOADM automatically, and the uplink optical signals, originally, from each set of BS-groups can be transmitted back to the $\mathrm{CO}$ end along the opposite direction of the downlink signal-routing path. In this way, the interference caused by fiber breakage can be avoided immediately, and the entire transport system can be reconnected to ensure the quality of network services. Our experimental results prove that the overall transmission performances are similar to those under normal circumstances.
\end{abstract}

Keywords: disconnection protection; optical add/drop multiplexer; radio over fiber; wavelength division multiplexing

\section{Introduction}

Various applications, such as virtual reality and $4 \mathrm{~K}$ video on-demand, increase the bandwidth demand of wireless communications, and radio over fiber (RoF) transmission systems are, therefore, being developed to meet the rapidly increasing demand [1-8]. With the upgrading of wireless broadband networks, such as from $4 \mathrm{G}$ networks to $5 \mathrm{G}$ networks, the signal coverage range of each base station (BS) will be reduced greatly and the density of the BS distribution will be increased significantly in metropolitan areas [9-13]. However, in a tree-topology network structure, the number of BSs that can be connected to each feeder fiber is limited to between 64 and 128 ( $2^{\mathrm{x}}$ to) $[14,15]$. Embedding wavelength division multiplexing (WDM) technique into time-division multiplexing (TDM) based treetopology network will be a suitable solution for overcoming this limitation [16]. If an optical multiplexing/de-multiplexing component, such as an arrayed waveguide grating (AWG), is added into the remote node (RN) of the tree-topology RoF transport systems to route different wavelengths of light carriers to different feeder fibers and significantly increase the number of BSs that can be served per feeder fiber $[17,18]$. The main drawbacks of such hybrid architecture are the limited scalability and flexibility of extending or reorganizing AWG's optical routing characteristics. 
In parallel with utilizing AWGs to form the hybrid TDM and WDM RoF network mentioned above, some scholars have proposed forming hybrid TDM and WDM RoF networks with better flexibility by utilizing an integrated ring and tree structure network instead of using only a tree structure [18-20]. Such hybrid network architecture provides a potential self-disconnection repair capability that can overcome unexpected fiber-link failure by using an optical add/drop multiplexer (OADM) to bridge optical wavelengths transmitted between the ring and tree topology. Each OADM node can utilize an alternate fiber loop for signal transmissions to avoid breakpoints to ensure that users will not experience any usage problems during fiber line repair, and the original bandwidth connection can also be maintained [21,22]. Nevertheless, a traditional OADM can only direct and route the downstream and upstream optical signals in the same direction of the ring-fiber link. Additional optical switches (SWs) are required to be embedded into each OADM node to form a bidirectional OADM (BOADM) and a spare ring-fiber link is necessary to achieve self-disconnection repair capabilities [23,24]. If the abovementioned two-wire BOADM can be changed to a single-line BOADM (SBOADM), then the entire ring network architecture only requires a single fiber ring structure to achieve the same self-disconnection protection effect. Therefore, good performance can be achieved in terms of system complexity, fiber laying costs, and logistics maintenance. Recently, some experts and scholars have proposed the use of SWs, array waveguides, optical circulators, and optical amplifiers, etc., to construct SBOADMs and utilize such SBOADMs to accomplish bidirectional WDM ring networks $[25,26]$. Such SBOADMs can utilize a single ring fiber link to achieve bidirectional transmissions properly. However, the SWs and optical amplifiers in such SBOADM are not passive components and require additional power supply to function properly. This scenario is not ideal in terms of system cost, architecture scalability, and system management and maintenance. Thus, to optimize and simplify the structure of the published SBOADMs, we have developed a new generation SBOADM to accomplish bi-directional add/drop multiplexing functionality by utilizing four passive optical circulators (OCs) and only two sets of fiber Bragg gratings (FBGs) [27]. The new generation SBOADM can always drop down the desired downstream signals whether the signals are injected into the SBOADM in each direction and can upload and send back the upstream signals via the reversed optical pathway of the downstream signals. Not any active device can be utilized inside this new generation SBOADM; to extend the application of the passive SBOADM, a hybrid ring- and tree-topology RoF transmission system with self-disconnection protection that utilized the new generation SBOADM for hybrid TDM and WDM RoF transport systems was proposed and verified. Comparing to the OADM-, BOADM- or published SBOADM-based WDM transport systems, no additional backup fiber loop, SW or any other active device is deployed among the CO and BSs. This means that no power supply is enquired in the passive fiber loop to accomplish the data transmission among the $\mathrm{CO}$ and BSs. The operating expense can be reduced significantly. Our experimental results prove that no complicated network management is required to accomplish the quality of service when a fiber-link failure occurs in any point of the transmission path. The system manager can simply adjust the state of an SW deployed inside the CO to recover all broken network connections.

\section{Experimental Setup}

A hybrid ring- and tree-topology RoF transmission system with self-disconnection protection is presented in Figure 1. The transmission system will first connect the CO with each SBOADM through a main ring-fiber link and then bridge each SBOADM with a BS-Group in the tree structure topology. The RF signal in the experiment is generated by modulating a 1.25 Gbps unipolar pseudo-random binary sequence (PRBS, generated by Anritsu MP1632a) with a $10 \mathrm{GHz}$ sinusoidal signal. The downstream RoF signal in CO is simulated by the modulated the $1.25 \mathrm{Gbps} / 10 \mathrm{GHz}$ signal with a dedicated optical carrier via Mach Zender Modulators (MZMs). The optical carriers for BS-Group1 to BS-Group4 were set at 1547.4, 1549.06, 1550.67 and $1552.19 \mathrm{~nm}$, respectively (the parameters are shown, 
in Table 1, in detail). These modulated RoF signals are consequently multiplexed by an AWG, amplified by an erbium-doped fiber amplifier (EDFA), and fed into the input port one of a $2 \times 2 \mathrm{SW}$, which is in a parallel state, before being injected into the downstream link. Each RoF signal will be routed out of the SW output port1 with $15 \mathrm{dBm}$ optical power and delivered sequentially through a series of single-mode fiber (SMFs) to SBOADM1 to SBOADM4. The distance among each SBOADM was set to $1 \mathrm{~km}$ (SMF2, SMF3, SMF4) and the distance between the CO and the SBOADM1 and the CO and SBOADM4 were set to $26 \mathrm{~km}$ (SMF1 and SMF5) to simplify the experimental setup. When the RoF signals enter each SBOADM, only a specific RoF signal is intercepted at the add/drop port and routed to the connected BS-Group, while the other RoF signals penetrate. The detailed routing pass way of the optical signals inside the SBOADM is indicated in the inset of Figure 1. Ideally, the desired RoF signal should be dropped by the SBOADM completely. However, the FBGs utilized inside the SBOADM cannot produce $100 \%$ reflection. The residual signal will also penetrate the SBOADM and moved forward to the next SBOADM, along with the remained RoF signals. The I/O_P2 of the last SBOADM (SBOADM4) will still have some downlink RoF signals' energy residue and will further inject into output port 2 of the $2 \times 2$ SW in the CO after penetrating SMF5 and a $2 \times 1$ optical coupler. These residual signals will be routed out of input port 2 of the SW and then routed back to the other input of the $2 \times 1$ optical coupler, and repeatedly injected into the SW. As a result, these amounts of residual signal energy will continue to be consumed in the process. When each dedicated downstream RoF signal is dropped by an SBOADM, a $2 \times 16$ optical splitter connected with the two add/drop ports of the SBOADM will distribute the RoF signal to each BS in the connected BS-Group. The downlink RoF signal in a BS will first be routed through an $\mathrm{OC}$ to a photodetector (PD) in the receiving unit and restored to a $1.25 \mathrm{Gbps} / 10 \mathrm{GHz} \mathrm{RF}$ signal. The RF signal is then increased, the frequency down-converted, the redundancy noise filtered and analyzed by an oscilloscope (HP83480a), bit error rate analyzer (Anritsu MP1632a), and electro-spectrum analyzer (thinkRF R5500).

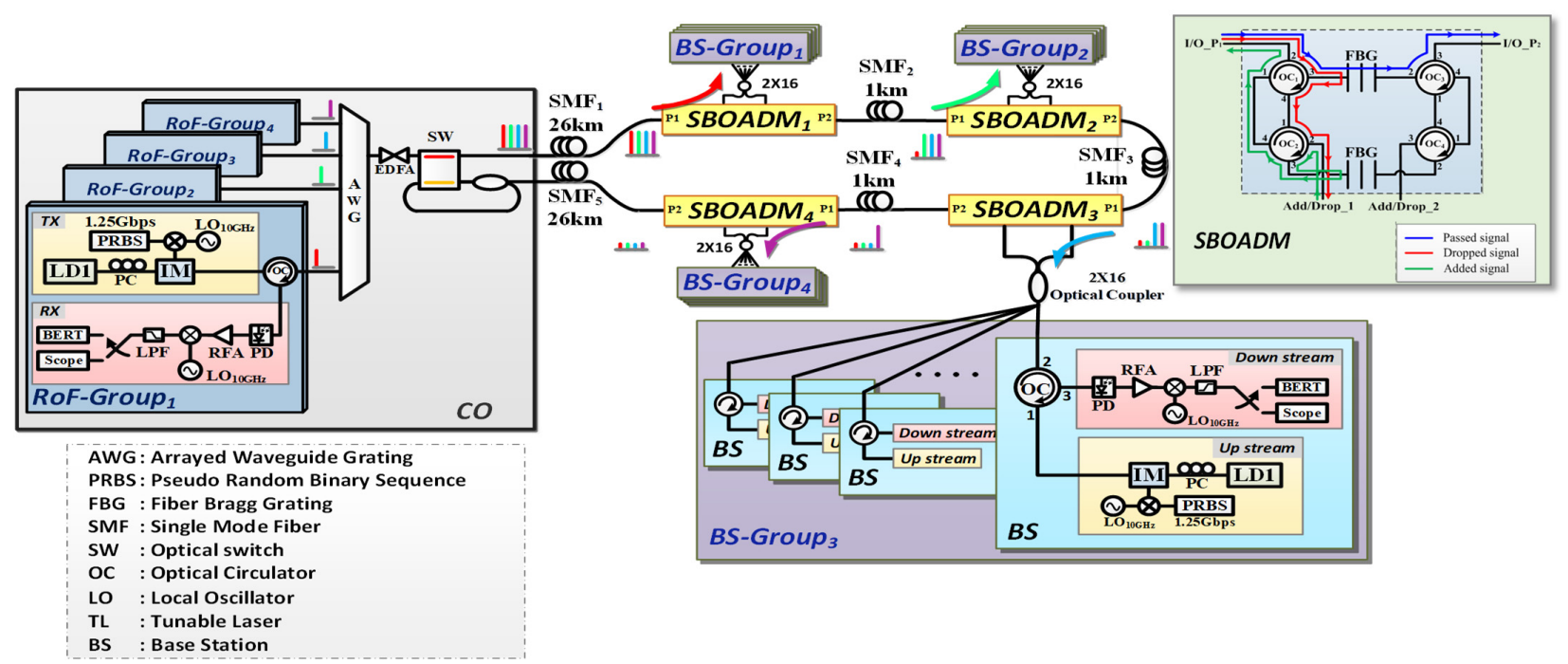

Figure 1. Hybrid ring- and tree-topology RoF transmission system with self-disconnection protection.

In the transmission part of the uplink signals, the uplink optical carrier wavelength used by each BS unit can be uploaded successfully to the transmission system via SBOADM by simply being in the FBG reflective wavelength range corresponding to the SBOADM. However, the uplink optical carrier wavelength of each BS is consistent with the downlink optical carrier wavelength to improve the usage efficiency of light wavelengths. Similar to the downstream transmission, the uplink $1.25 \mathrm{Gbps} / 10 \mathrm{GHz}$ RF signal of each BS is modulated with the uplink optical carrier via MZM. Consequently, the uplink signal in each BS is routed through the OC to the $16 \times 2$ optical coupler and injected simultaneously 
into the Add/Drop_1 and Add/Drop_2 of the connected SBOADM. The signals injected into the Add/Drop_2 of the SBOADM will be routed to the I/O_P2 of the SBOADM and moved forward to the $\mathrm{CO}$ along with the residual downlink light signals. Uplink RoF signal energy will continue to be consumed in the process, along with the residual downlink RoF signal energy. In parallel, the RoF signal injected into the Add/Drop_1 of the SBOADM will be routed to the I/O_P1 of the SBOADM and routed back to the CO along the reversed transmission pathway of the downlink signals. The uplink optical signal in the $\mathrm{CO}$ end is converted back to the electrical domain and verified by instruments.

Table 1. System parameters.

\begin{tabular}{ccccc}
\hline $\begin{array}{c}\text { Optical Carriers } \\
\text { (Santec TSL210) }\end{array}$ & $\begin{array}{c}\text { RF Signal (Anritsu } \\
\text { MG3697c) }\end{array}$ & $\begin{array}{c}\text { Baseband Signal } \\
\text { (Anritsu MP1632a) }\end{array}$ & Modulation & $\begin{array}{c}\text { Optical Spectrum } \\
\text { Analyzer }\end{array}$ \\
\hline $\begin{array}{l}1547.40 \mathrm{~nm} \\
1549.06 \mathrm{~nm}\end{array}$ & $10 \mathrm{GHz}$ & $1.25 \mathrm{Gbps}$ (PRBS) & Mach Zender & Anritsu MS9740A \\
$1550.67 \mathrm{~nm}$ & & & & \\
$1552.19 \mathrm{~nm}$ & & & & \\
\hline
\end{tabular}

\section{Experimental Results and Discussion}

In this experiment, the optical powers of the downlink optical signals before being injected into the $26 \mathrm{~km} \mathrm{SMF1}$, and the uplink optical signals before being injected into the $16 \times 2$ optical coupler, are 15 and $5 \mathrm{dBm}$, respectively. Based on the characteristics of the FBGs within each SBOADM, the $1547.4 \mathrm{~nm}$ light carrier is intercepted into the BS-Group1 when the downlink signals are transmitted to SBOADM1 through the 26-km SMF1. The remaining 1549.06, 1550.67 and $1552.19 \mathrm{~nm}$ optical signals are captured by SBOADM2, SBOADM3, and SBOADM4 in sequence and then sent to BSs in BS-Group2, BS-Group3, and BS-Group4, respectively. The wavelength spacing between each two adjacent optical signals is $1.66,1.61$, and $1.52 \mathrm{~nm}$. Since these four light sources have sufficiently wavelengthspaced (larger than 19-GHz frequency-spaced) from its neighbor, the interference caused in the main channel from the neighboring channel is insignificant [28]. Figure 2a-d shows the spectra of four downlink RoF signals measured as they penetrate from SBOADM1 to SBOADM4. In this experiment, the reflectivity of each FBG inside SBOADMs is not identical but is roughly around $97 \%$. Thus, the power difference between the captured light carrier and the penetrated light carrier was inconsistent after penetrating through each SBOADM. The power differences are approximately 14.6, 13.9 and $16.1 \mathrm{~dB}$ after penetrating SBOADM1, SBOADM2, and SBOADM3, respectively. After penetrating SBOADM4, residual optical signals, as shown in Figure 2d, can be considered as noise for the entire transmission system, but this noise can be consumed naturally by the $2 \times 1$ optical coupler and SW in CO after passed through the SMF5.

When the downlink signal is extracted by each SBOADM and injected into each BS-Group, the spectrum measured in front of the PDs of BSs at the BS-Group1 to BSGroup4 are shown in Figure 3a-d, respectively. In the four groups, the optical signal power levels to be received were $-7.09,-8.94,-10.81$ and $-12.67 \mathrm{dBm}$, respectively. The power difference between the two adjacent BS-Groups is roughly $1.85 \mathrm{~dB}$, which is approximately equivalent to the insertion loss when the signal penetrates from I/O_P1 of SBOADM to I/O_P2. Although parts of the untargeted signals are also dropped and directed to the BSs, their power levels are much lower than the targeted signal. Comparing the peak power difference among the targeted optical signal and the untargeted residual signals, the optical signal-to-noise power ratios (OSNRs) for the four BS-Groups were 29.1, 28.6, 32.2 and $31.67 \mathrm{~dB}$, respectively. When the four sets of downlink optical signals are received by the PD of each BS, they can be successfully detected and the obtained RF signals can be converted back to baseband format. The electro-spectrum and eye diagram of the frequency down-converted downlink RoF signals received by BS-Group1 to BS-Group4 are indicated in Figure $4 \mathrm{a}-\mathrm{d}$. The frequency down-converted $1.25 \mathrm{Gbps}$ NRZ signal is clearly presented 
in the baseband area. This means that all four sets of RoF signals are received successfully by each BS-Group. Besides, when the received NRZ sequence is fed into an oscilloscope, open and clear eye diagrams are also obtained. This means that very little intersymbol interference (ISI) is presented and the interference caused in the main channel from the neighboring channel is ignorable.

(a)

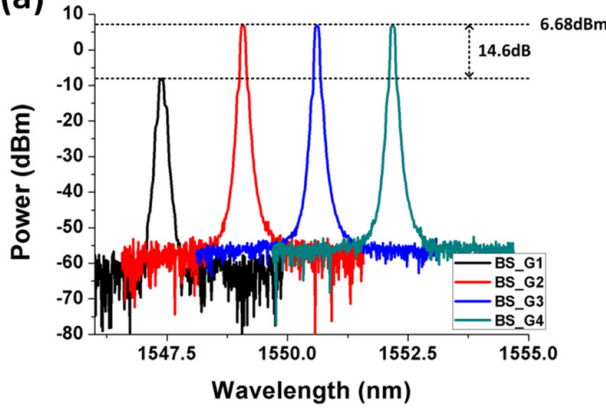

(c)

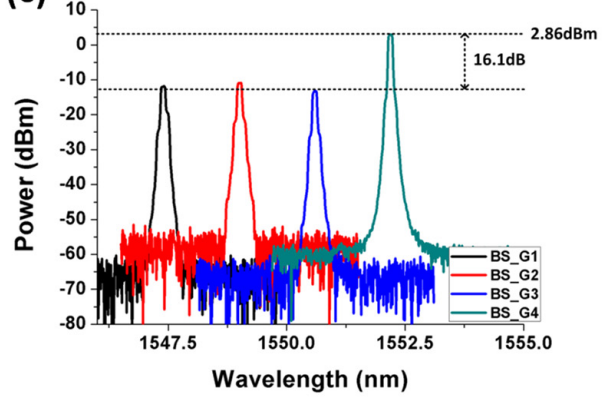

(b) 10

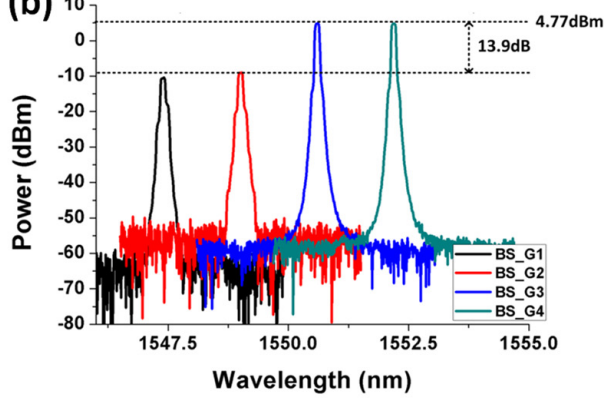

(d)

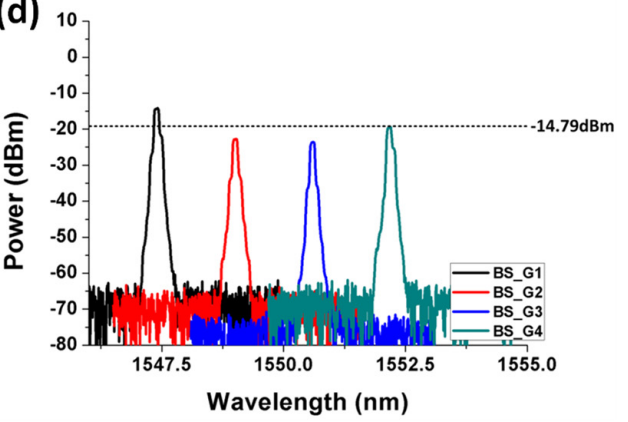

Figure 2. Spectrum of downlink light signals after penetrating (a) SBOADM1, (b) SBOADM2, (c) SBOADM3 and (d) SBOADM4.
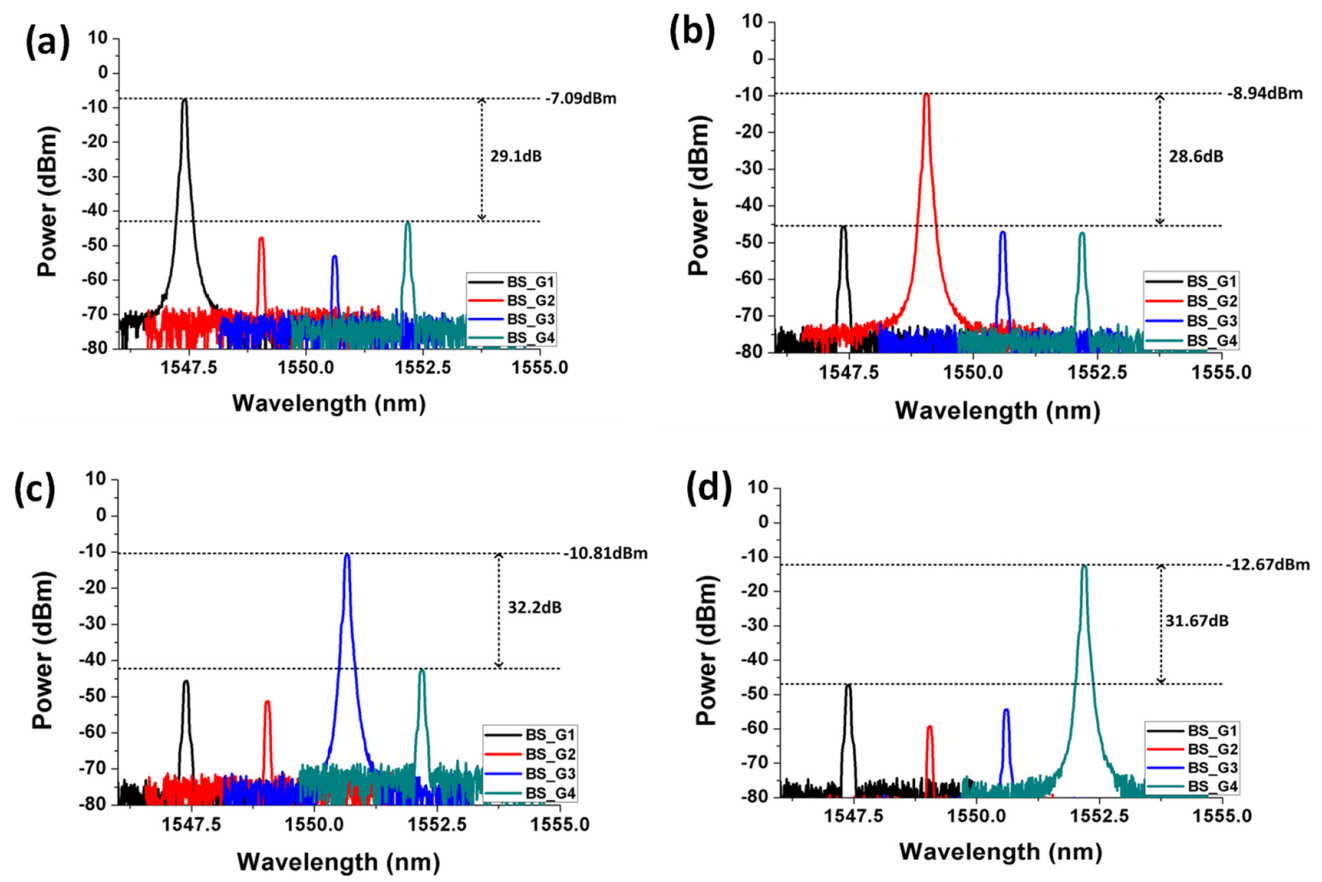

Figure 3. Spectrum measured in front of the PDs of BSs at the (a) BS-Group1, (b) BS-Group2, (c) BS-Group3 and (d) BS-Group4. 

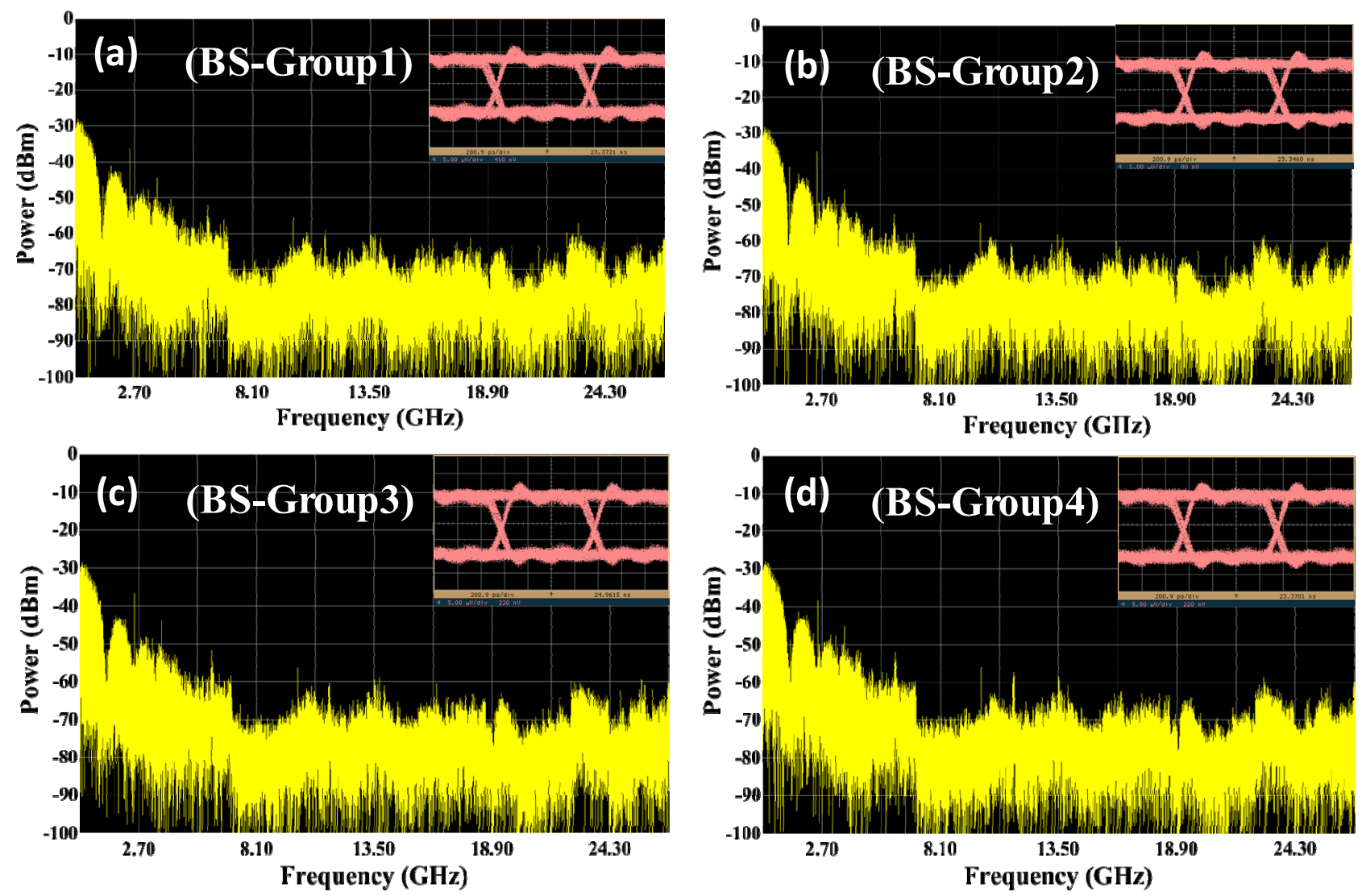

Figure 4. Electro-spectrum and eye diagram of frequency down-converted downlink RoF signals received by (a) BS-Group1, (b) BS-Group2, (c) BS-Group3 and (d) BS-Group4.

In terms of uplink signal transmission, the optical signals generated from each BS are injected into the Add/Drop_1 and Add/Drop_2 of the connected SBOADM after passing through a $16 \times 2$ optical coupler. As discussed in the previous section, the uplink optical signals routed to the Add/Drop_2 of the SBOADM will be transmitted back to the $\mathrm{CO}$ along the $\mathrm{CW}$ direction of the main ring fiber link and consumed completely at the SW in the CO. By contrast, the uplink optical signals injected into the Add/Drop_1 of SBOADM will be routed to the I/O_P1 of the SBOADM and be sent back to the CO along the CCW direction of the main ring fiber link. Given that each SBOADM only drops down a dedicated optical signal to the connected BS-Group during this routing process, all uplink optical signals can successfully penetrate the other SBOADMs and pass along the SMF1 to the CO end when transmitted in the main ring-fiber link. The optical spectrum of each uplink signal received at the CO end of each BS-Group is overlapped and shown in Figure 5. The power levels of the uplink optical signals generated from the BSs at the BS-Group1, BS-Group2, BS-Group3, and BS-Group4 were $-7.06,-10.7,-14.38$, and $-18 \mathrm{dBm}$, respectively. The power differences between the two adjacent sets of uplink optical signals were $3.64,3.68$, and $3.62 \mathrm{~dB}$. These values are roughly equivalent to the insertion loss ( $3.46 \mathrm{~dB}$ ) when the signal penetrates from I/O_P2 of the SBOADM to I/O_P1. Similarly to the downlink transmissions, all four sets of the uplink RoF signals were also received successfully and converted back to baseband format. Figure 6a-d shows that the transmitted signals are obtained clearly in the spectrum diagrams and the measured eye diagrams are open and clear. 


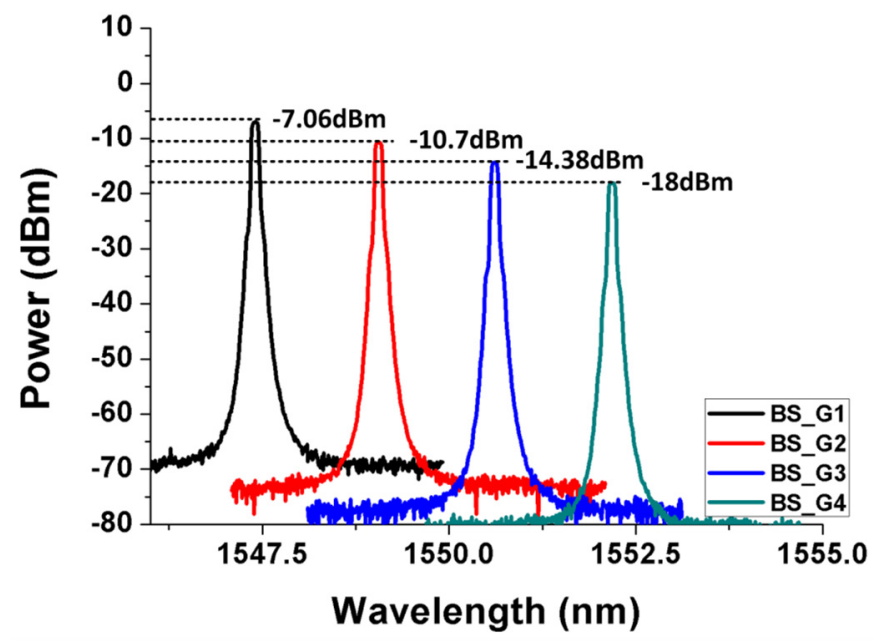

Figure 5. Overlapped uplink optical spectra measured at the CO end of each BS-Group.
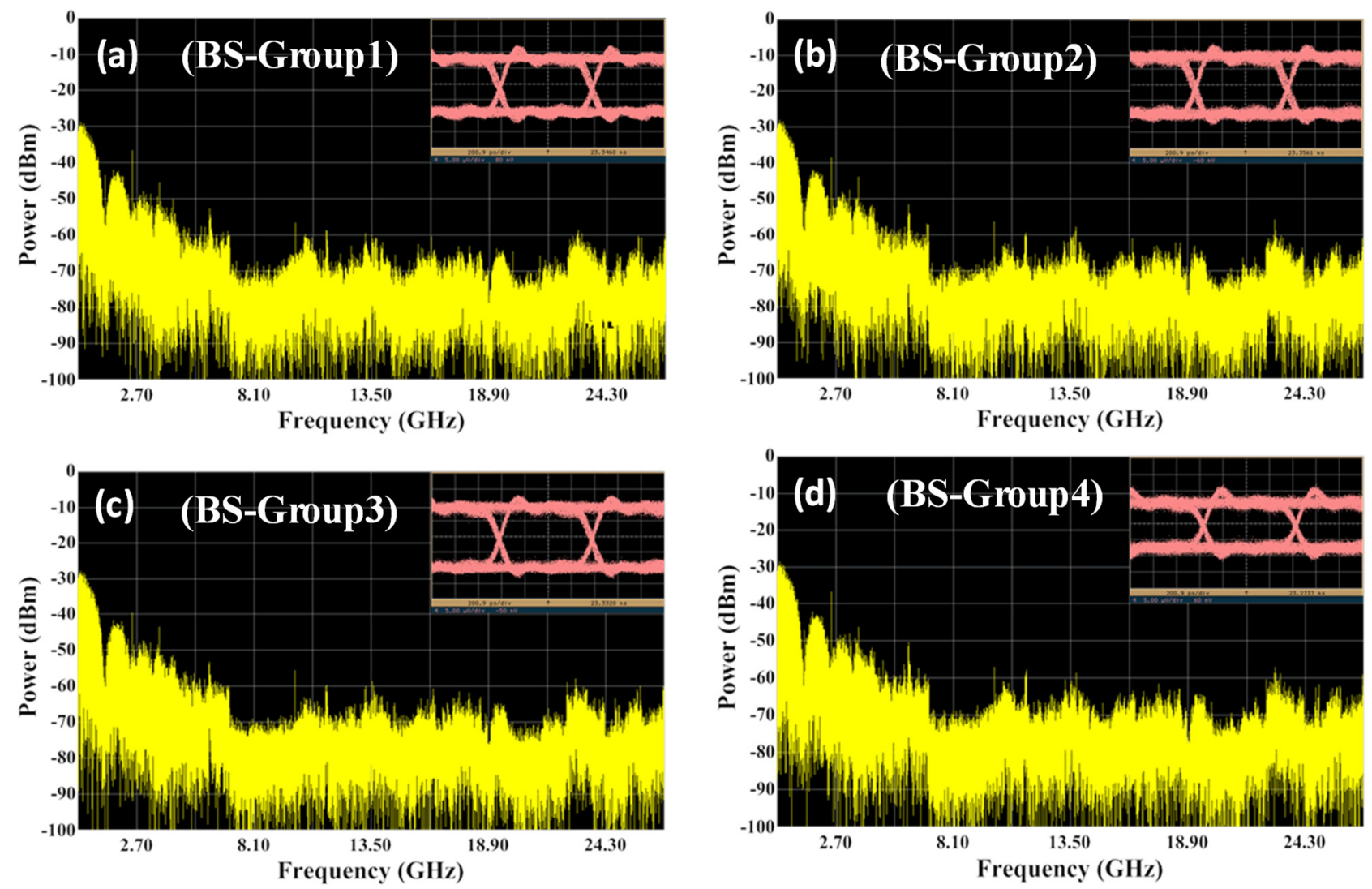

Figure 6. Electro-spectrum and eye diagram of frequency down-converted uplink RoF signals received at the CO end of (a) BS-Group1, (b) BS-Group2, (c) BS-Group3 and (d) BS-Group4.

In addition to observing the spectra and eye diagrams, the raw bit error rate (BER) performances for the BSs in BS-Group1 to BS-Group4 (indicated as BS-G1, BS-G2, BS-G3, and BS-G4) in the back-to-back (B2B) and downlink transmissions scenarios and in the B2B and uplink transmissions scenarios are illustrated in Figure 7a,b. Instead of estimating the BER value based on a few measured moments of the probability density function, such as Q-factor or error-vector magnitude (EVM), the raw BER is the ultimate quality measure in optical communication links [29]. To obtain the raw BER values, the down-frequency RF signals were analyzed by an Anritsu MP1632a and their BER values are calculated by dividing the number of errors by the total number of bits sent. It is clear that each set 
of signals can enjoy an error rate lower than $10^{-9}$ when the receiver powers are larger than -21.5 to $-21.2 \mathrm{dBm}$ for downlink transmissions and around -19.5 to $-19 \mathrm{dBm}$ for uplink transmissions. Compared with the B2B scenario, the power penalty of the four sets of signals was all less than $0.5 \mathrm{~dB}$ for the downlink and uplink transmissions. The clear eye diagram and error-free transmissions provide considerable evidence of the advanced transmission performance of the proposed system.
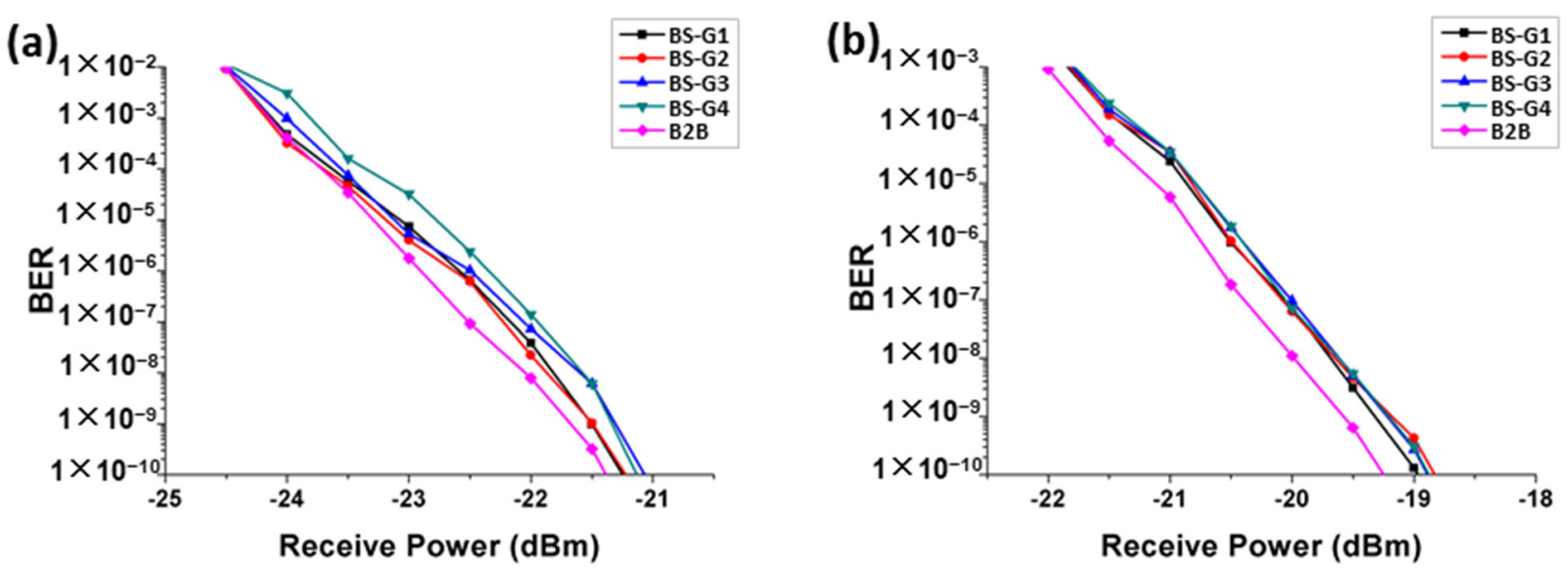

Figure 7. BER performances for the BSs in BS-Group1 to BS-Group4 in (a) B2B and downlink transmissions scenarios as well as in (b) B2B and uplink transmissions scenarios.

In verifying the breakpoint protection function of the proposed transport system, we assume that the SMF3 located between the SBOADM2 and SBOADM3 is interrupted for any reason. As a result, the SBOADM3 and the SBOADM4 will lose connections with the $\mathrm{CO}$ end. In this case, the interrupted BS-Groups can be reconnected to the $\mathrm{CO}$ end by simply changing the SW in the CO end from a parallel-bridge state to a cross-bridge state. Figure 8 shows that the downlink optical signals in this case are separated into two paths through a $1 \times 2$ optical splitter after passing through output port 2 of the SW. One set of downlink optical signals is routed back to the input port2 of the SW and then passed through the SW in the cross-bridge state and the SMF1 to the SBOADM1 and the SBOADM2 in a CW direction (the route path under normal conditions). The other set of downlink optical signals emitted from the $1 \times 2$ optical splitter is routed to the SMF5 and directed to the SBOADM4 and SBOADM3 in a CCW direction. With the bi-directional transmission characteristics of the new-generation SBOADM, the target optical signals can still be intercepted at the corresponding BS-Group as the downlink optical signal is injected into the SBOADM via I/O_P1 or the I/O_P2. Meanwhile, the non-target optical signals can penetrate the SBOADM directly. When the optical signals are fed into the SBOADM via I/O_P2, the detailed routing pass way of the optical signals inside the SBOADM is indicated in the inset of Figure 8.

Figure 9a-d show the spectrum measured when the downlink optical signal penetrates the SBOADM1 and SBOADM2 in the CW direction and penetrates the SBOADM3 and SBOADM4 in the CCW direction when a breakpoint occurs. Figure 10a-d shows the spectra measured at the receiving end of BS when the downlink optical signal is captured by SBOADM to the BS in BS-Group1 to BS-Group4 when a breakpoint occurs. The spectra of the signal penetrating SBOADM1 and SBOADM2 are roughly similar to the data measured under the condition of no breakpoints (Figure 2). However, each light carrier will have extra attenuation because of the additional passing of the $1 \times 2$ optical splitter and SW during the transmission process. In the spectrum penetrating SBOADM3 and SBOADM4, the downlink signal is injected into the SBOADMs through the I/O_P2 of the SBOADMs. The results of the initial experiment on SBOADM show that the insertion loss of penetrating light signals was approximately $3.46 \mathrm{~dB}$, which was $1.8 \mathrm{~dB}$ more than when injected through I/O_P1. Therefore, the maximum power of the non-target optical signal in Figure $9 \mathrm{~d}$ is 
lower than the value in Figure 9a. In addition, a comparison of the non-target optical signal power that penetrates SBOADM4 and SBOADM3 shows that the difference between these two values was approximately $3.7 \mathrm{~dB}(1.92-(-1.78)=3.70 \mathrm{~dB})$. Figure $10 \mathrm{a}-\mathrm{d}$ also shows that the power levels of the target optical signal were $28 \mathrm{~dB}$ to $32 \mathrm{~dB}$ higher than that of the non-target optical signal when each group of downlink optical signals is intercepted by each BS-Group. Thus, the transmission of the RoF signal can be received and restored successfully to the electrical domain.

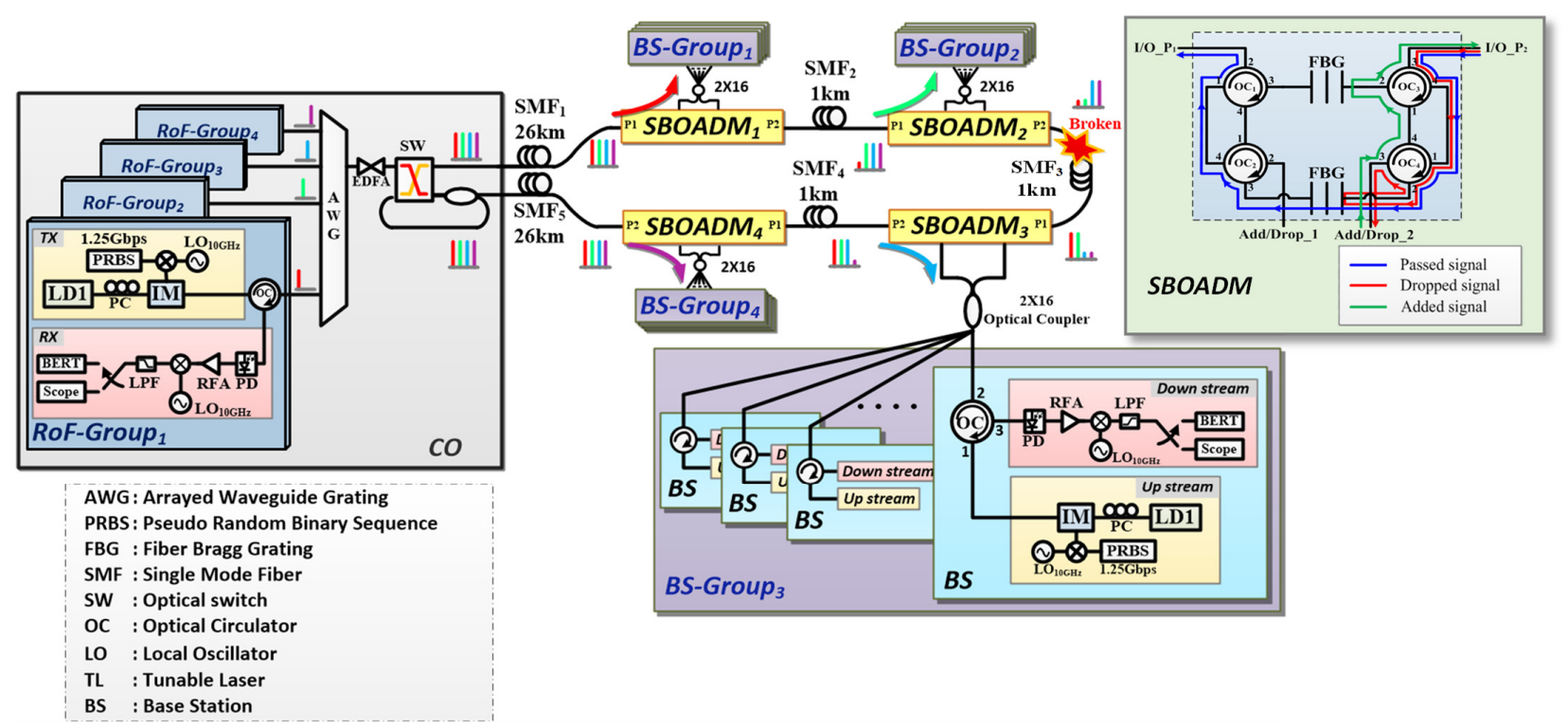

Figure 8. Diagram of the routing of downlink optical signal in the hybrid ring- and tree-topology RoF transmission system when breakpoints occur.
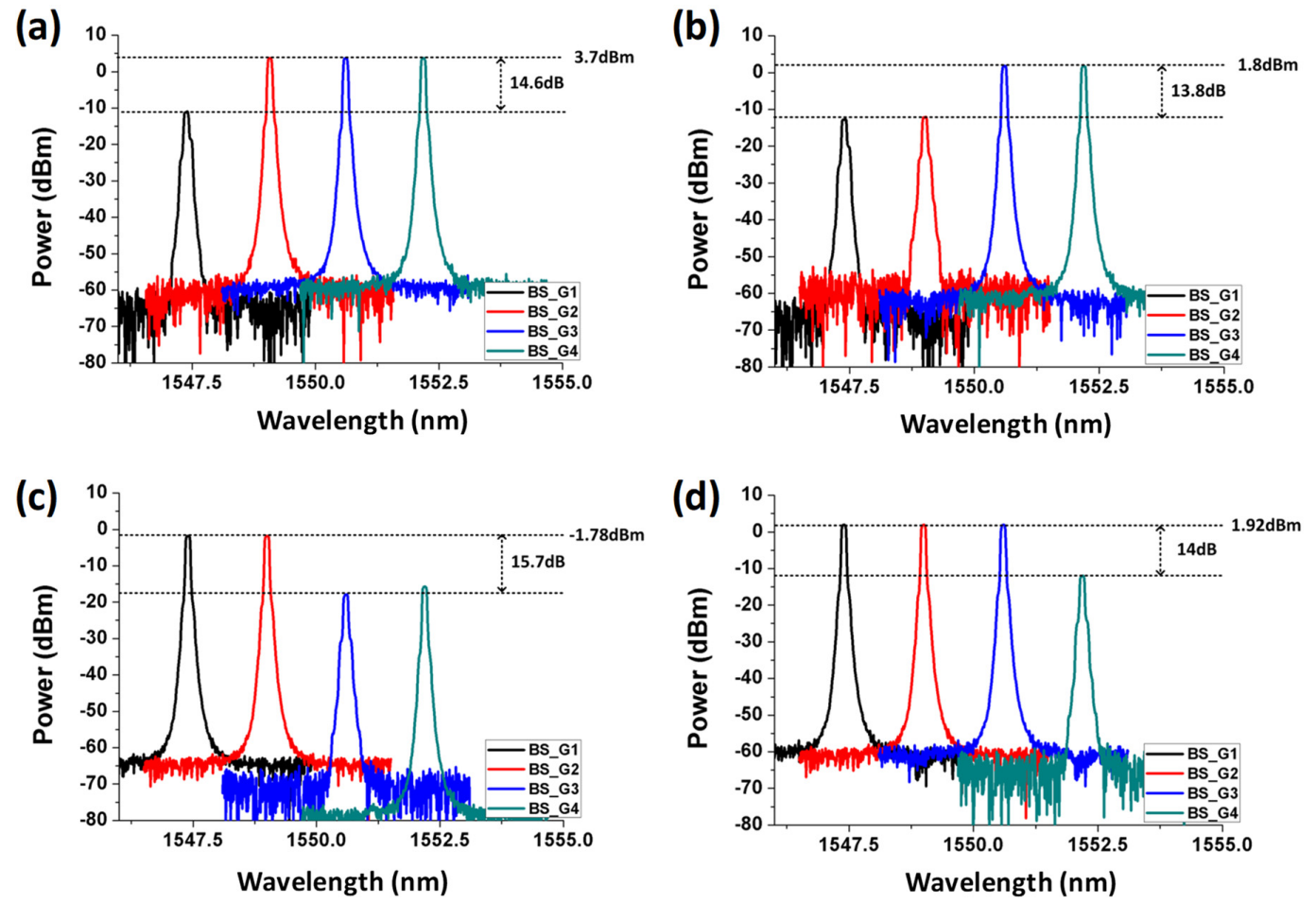

Figure 9. The spectrum measured when the downlink optical signal penetrates (a) SBOADM1 and (b) SBOADM2 in CW direction and penetrates (c) SBOADM3 and (d) SBOADM4 in the CCW direction when a breakpoint occurs. 

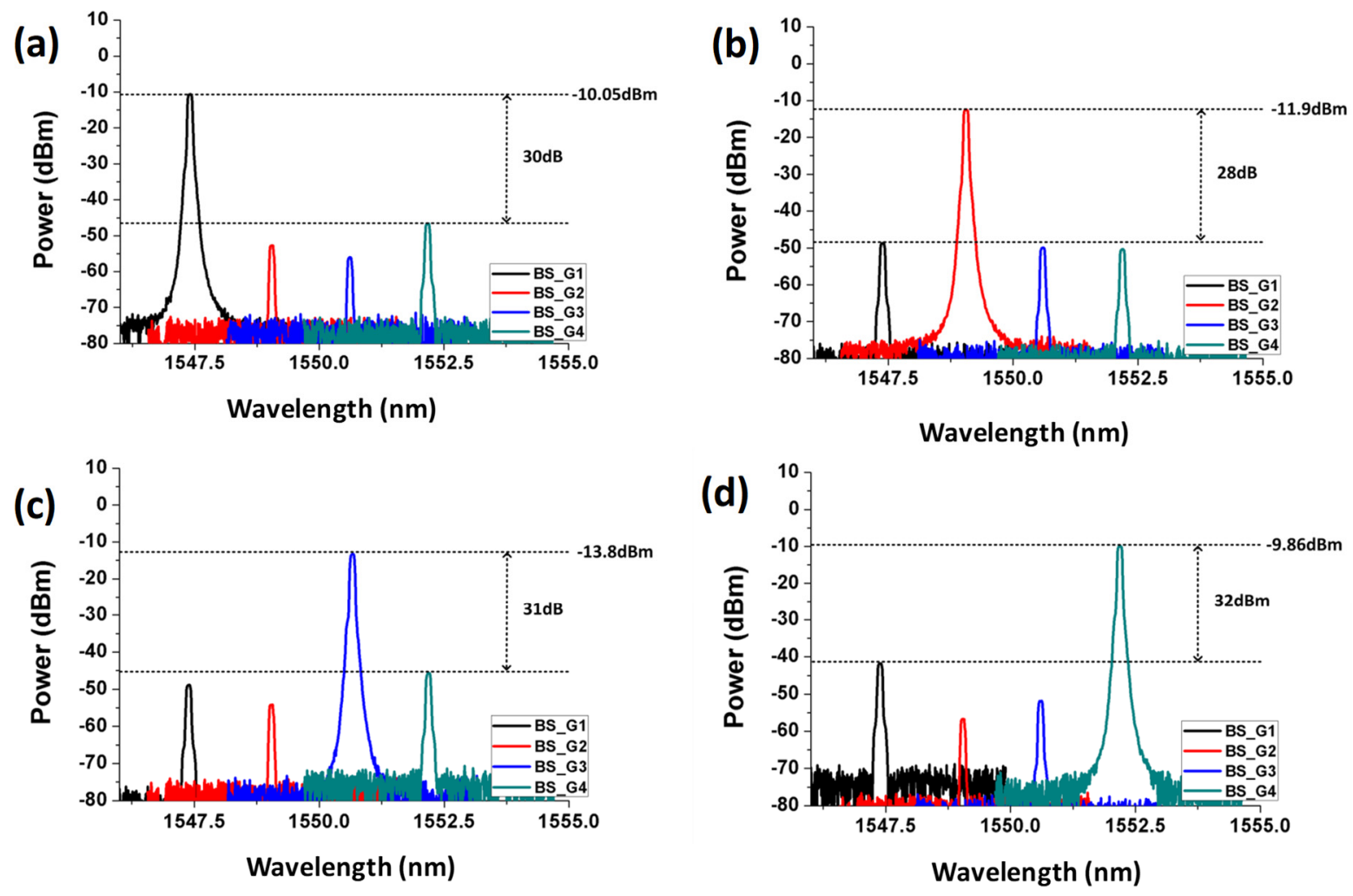

Figure 10. Spectrum measured at the receiving end of BS when the downlink optical signal is captured by SBOADM to the BS in (a) BS-Group1, (b) BS-Group2, (c) BS-Group3 and (d) BS-Group4 when a breakpoint occurs.

In the transmission portion of the uplink signals, the uplink signals of each BS are routed to the Add/Drop_1 and Add/Drop_2 of the connected SBOADM at the same time and passed in the $\mathrm{CW}$ and $\mathrm{CCW}$ directions to the main ring-fiber link. The $\mathrm{CW}$ transmission of optical uplink signals via SBOADM1 and SBOADM2 and the CCW transmission of optical uplink signals via SBOADM3 and SBOADM4 are blocked by breakpoints from returning to the $\mathrm{CO}$ end. By contrast, the uplink optical signal transmitted CCW through SBOADM1 and SBOADM2 and those transmitted CW through SBOADM3 and SBOADM4 can be transmitted successfully to the $\mathrm{CO}$ end. The optical spectra of the uplink signals measured at the $\mathrm{CO}$ end are overlapped and presented in Figure 11, and the error rate graphs of each BS-Group measured at the CO end are shown in Figure 12. The power levels of the received uplink optical signals generated from the BSs at BS-Group1, BSGroup2, BS-Group3, and BS-Group4 were $-10.5,-13.8,-12.7$ and $-10.9 \mathrm{dBm}$, respectively. Compared with normal conditions, the uplink optical signals transmitted from BS-Group1 and BS-Group2 suffer slightly larger power attenuation in the transmission process because these signals have to pass through an additional $1 \times 2$ optical splitter. Nevertheless, the transmission losses of the uplink optical signals transmitted from BS-Group3 and BSGroup4 are reduced because the routing path is shorter than that in normal conditions. The overall results presented in the downlink and uplink transmissions when a breakpoint occurs were roughly similar to those under normal conditions except for the slight variation in power. These results demonstrate that the proposed hybrid ring- and tree-topology RoF transmission system can integrate TDM and WDM techniques properly into a signal network architecture to increase the supportable BS number and provide robust fiber-link disconnect protection to ensure quality of services. 


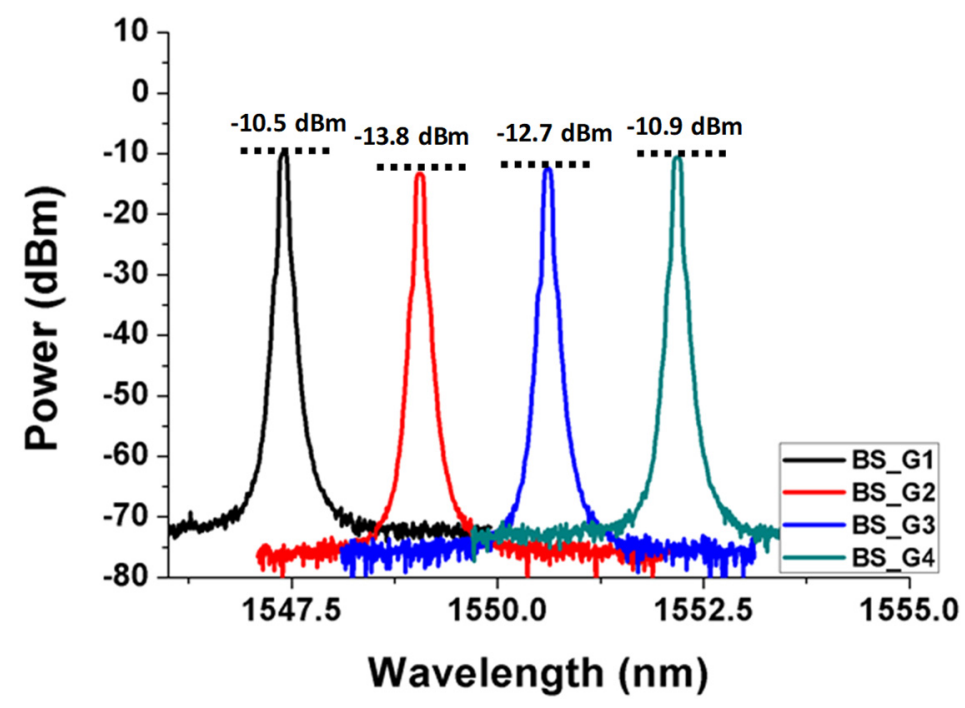

Figure 11. The optical spectra of uplink signals are measured at the $\mathrm{CO}$ end when a breakpoint occurs.
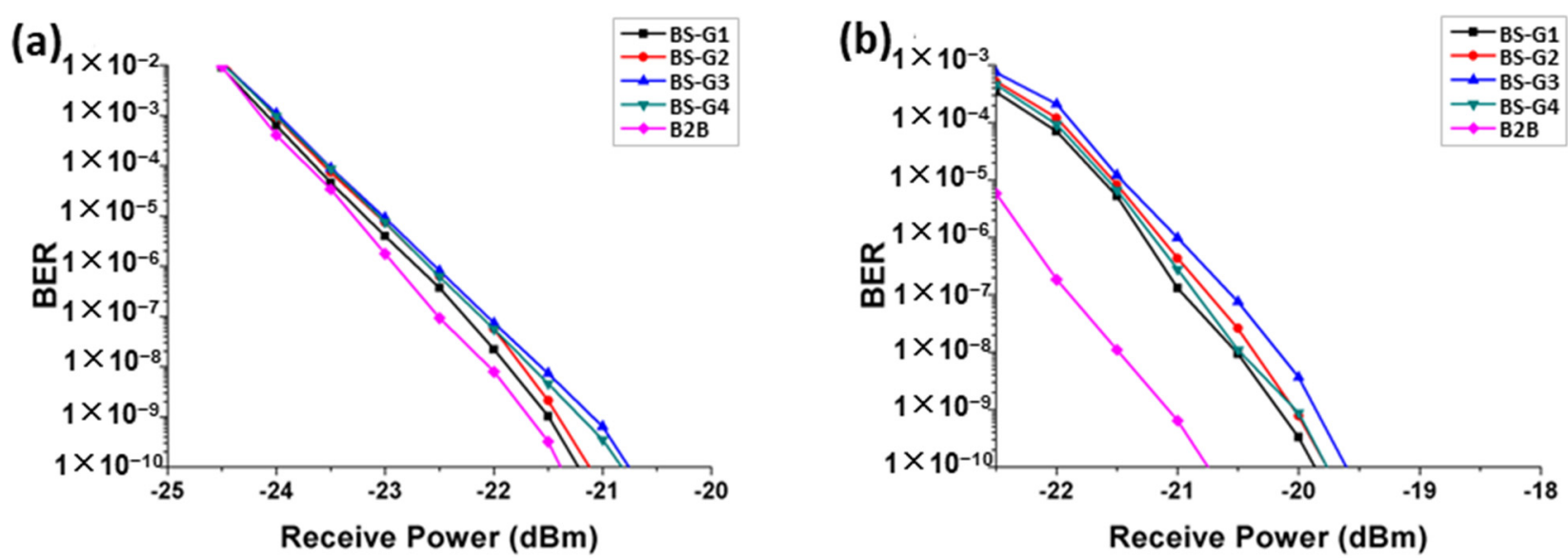

Figure 12. (a) Downlink RoF signal error rate performance measured in BS-Group1, BS-Group2, BS-Group3, BS-Group4, and Back-to-Back when a breakpoint occurs. (b) Uplink RoF signal error rate of BS-Group1, BS-Group2, BS-Group3, BS-Group4, and Back-to-Back measured at the $\mathrm{CO}$ end when a breakpoint occurs.

\section{Conclusions}

A hybrid ring- and tree-topology RoF transmission system with self-disconnection protection was proposed, to integrate TDM and WDM techniques and increase the number of supportable BS in a signal-network architecture. The quality of service still can be ensured when the main ring-fiber link of the transmission system is interrupted for any reason by utilizing new-generation SBOADMs properly to bridge signal transmissions in the proposed architecture. The system operator can recover the broken connections quickly by transforming the operation state of an SW in the $\mathrm{CO}$ to allow the downlink signals to be transmitted in the $\mathrm{CW}$ and $\mathrm{CCW}$ directions of the main ring-fiber link at the same time. The employed SBOADMs with two-way transmission characteristics will drop down the desired RoF signals automatically into the connected BS-Group. The uplink optical signal emitted from each BS can also be transmitted back to the $\mathrm{CO}$ end along the opposite direction of its downlink signal-routing path. In this way, the interference of the fiber breakpoint can be avoided immediately. Accordingly, the entire transmission system can be reconnected and the quality of network services will be further ensured. Our experimental results showed that the proposed hybrid ring- and tree-topology RoF transmission system can provide similar transmission performances in fiber breakpoint scenarios and in normal circumstances. 
Author Contributions: C.-Y.L. analyzed the data and started the manuscript. C.-H.C. conceptualized and supervised the research work and improved the manuscript for submission and publication. Z.-G.L. performed the experimental work. All authors have read and agreed to the published version of the manuscript.

Funding: This research was funded by the Ministry of Science and Technology of Taiwan (Grant No.: MOST 107-2221-E-415-009-, MOST 108-2221-E-415-023-, MOST 109-2636-E-305-003-, and MOST 110-2221-E-415-009-) and the University System of Taipei Joint Research Program under USTP-NTUTNTPU-110-01.

Institutional Review Board Statement: Not applicable.

Informed Consent Statement: Not applicable.

Conflicts of Interest: The authors declare that there is no conflict of interests regarding the publication of this paper.

\section{References}

1. Sengupta, D. ETIMC:Internet Is Moving from a Luxury to a Necessity. ETTelecom, 17 March 2017; p. 6.

2. Yeh, C.; Chow, C.; Chen, H.; Sung, J. Hybrid OFDM-based multi-band wireless and baseband signal transmission in PON access. Electron. Lett. 2012, 48, 390-392. [CrossRef]

3. Liu, D.; Tang, M.; Fu, S.; Shum, P. A long-reach WDM passive optical network enabling broadcasting service with centralized light source. Opt. Commun. 2012, 285, 433-438. [CrossRef]

4. Liu, C.; Wang, J.; Cheng, L.; Zhu, M.; Chang, G.-K. Key Microwave-Photonics Technologies for Next-Generation Cloud-Based Radio Access Networks. J. Light. Technol. 2014, 32, 3452-3460. [CrossRef]

5. Paredes-Páliz, D.F.; Royo, G.; Aznar, F.; Aldea, C.; Celma, S. Radio over Fiber: An Alternative Broadband Network Technology for IoT. Electronics 2020, 9, 1785. [CrossRef]

6. Al-Zubaidi, F.M.A.; López Cardona, J.D.; Montero, D.S.; Vázquez, C. Optically Powered Radio-Over-Fiber Systems in Support of 5G Cellular Networks and IoT. IEEE/OSA J. Lightwave Technol. 2021, 39, 4262-4269. [CrossRef]

7. Wang, J.; Jia, Z.; Campos, L.A.; Knittle, C.; Jia, S. Delta-Sigma Modulation for Next Generation Fronthaul Interface. J. Light. Technol. 2018, 37, 2838-2850. [CrossRef]

8. Castleford, D.; Nirmalathas, A.; Novak, D.; Tucker, R.S. Optical crosstalk in fiber-radio WDM networks. IEEE Trans. Microw. Theory Tech. 2001, 49, 2030-2035. [CrossRef]

9. Ranaweera, C.; Wong, E.; Nirmalathas, A.; Jayasundara, C.; Lim, C. 5G C-RAN With Optical Fronthaul: An Analysis From a Deployment Perspective. J. Light. Technol. 2017, 36, 2059-2068. [CrossRef]

10. Asha, D.S. A Comprehensive Review of Millimeter Wave Based Radio over Fiber for 5G Front Haul Transmissions. Indian J. Sci. Technol. 2021, 14, 86-100.

11. Hadi, M.; Awais, M.; Raza, M.; Khurshid, K.; Jung, H. Neural Network DPD for Aggrandizing SM-VCSEL-SSMF-Based Radio over Fiber Link Performance. Photonics 2021, 8, 19. [CrossRef]

12. Pandey, G.; Choudhary, A.; Dixit, A. Wavelength Division Multiplexed Radio Over Fiber Links for 5G Fronthaul Networks. IEEE J. Sel. Areas Commun. 2021, 39, 2789-2803. [CrossRef]

13. Li, G.; Deng, J.; Xin, S.; Huang, X.G. A Radio over Fiber System Compatible with 3G/4G/5G for Full Spectrum Access and Handover with Multi-Scenarios. J. Light. Technol. 2021. [CrossRef]

14. Luo, Y.; Zhou, X.; Effenberger, F.; Yan, X.; Peng, G.; Qian, Y.; Ma, Y. Time- and Wavelength-Division Multiplexed Passive Optical Network (TWDM-PON) for Next-Generation PON Stage 2 (NG-PON2). J. Light. Technol. 2012, 31, 587-593. [CrossRef]

15. Li, J.; Lee, K.-L.; Chan, C.A.; Anthapadmanabhan, N.P.; Dinh, N.; Vetter, P. Dynamic Power Management at the Access Node and Customer Premises in Point-to-Point and Time-Division Optical Access. IEEE J. Sel. Areas Commun. 2014, 32, 1575-1584. [CrossRef]

16. Abdalla, M.E.; Idrus, S.M.; Mohammad, A.B. Hybrid TDM-WDM 10G-PON for High Scalability Next Generation PON. In Proceedings of the IEEE 8th Conference on Industrial Electronics and Applications, Melbourne, VIC, Australia, 19-21 June 2013.

17. De Andrade, M.; Buttaboni, A.; Tornatore, M.; Boffi, P.; Martelli, P.; Pattavina, A. Optimization of Long-reach TDM/WDM Passive Optical Networks. In Proceedings of the 23rd Wireless and Optical Communication Conference, Newark, NJ, USA, 9-10 May 2014.

18. Feng, C.; Gan, C.; Gao, Z.; Wu, C. Novel WDM Access Network Featuring Self-healing Capability and Flexible Extensibility. In Proceedings of the 2015 International Conference on Electrical, Computer Engineering and Electronics, Jinan, China, 29-31 May 2015. [CrossRef]

19. Chow, C.W.; Yeh, C.H.; Sung, J.Y. Upgrading from TDM-PON to Signal-Remodulated WDM-PON with Rayleigh Backscattering Mitigation. In Proceedings of the OptoElectronics and Communications Conference and Photonics in Switching 2013, Kyoto, Japan, 30 June-4 July 2013. [CrossRef]

20. Urban, P.P.; Pluk, E.G.C.; De Laat, M.M.; Huijskens, F.F.; Khoe, G.G.-D.; Koonen, T.; De Waardt, H. 1.25-Gb/s Transmission Over an Access Network Link With Tunable OADM and a Reflective SOA. IEEE Photonics Technol. Lett. 2009, 21, 380-382. [CrossRef] 
21. Yang, B.; Chen, X.; Shen, C.; Huang, X.; Ma, Z.; Bei, J.; Li, M.; Zhu, Q.; Na, T. Smile OAN: A Long Reach Hybrid WDM/TDM Passive Optical Network for Next Generation Optical Access. In Proceedings of the Asia Communications and Photonics Conference 2016, Wuhan, China, 2-5 November 2016. [CrossRef]

22. Akhtar, A.; Turukhin, A.; Parsons, E.; Bakhshi, B.; Cardoso, J.; Vejas, M.; Savidis, H.; Derr, N.; Kovsh, D.; Golovchenko, E.; et al. First Field Demonstration of Fault Resilience in a Regional Undersea OADM Network. In Proceedings of the OFC/NFOEC, Los Angeles, CA, USA, 4-8 March 2012. [CrossRef]

23. Pires, J.J.O. Constraints on The Design of 2-Fiber Bi-Directional WDM Rings with Optical Multiplexer Section Protection. In Proceedings of the Digest of LEOS Summer Topical Meetings: Advanced Semiconductor Lasers and Applications/Ultraviolet and Blue Lasers and Their Applications/Ultralong Haul DWDM Transmission and Networking/WDM Compo, Copper Mountain, CO, USA, 30 July-1 August 2001.

24. Hu, X.; Chen, X.; Zhang, Z.; Wang, L.; Bei, J. Flexible ring-tree TWDM network architecture for next generation optical access network. In Proceedings of the 2014 23rd Wireless and Optical Communication Conference (WOCC), Newark, NJ, USA, 9-10 May 2014; pp. 1-3. [CrossRef]

25. Park, S.-B.; Lee, C.-H.; Kang, S.G.; Lee, S.B. Bidirectional WDM self-healing ring network for hub/remote nodes. IEEE Photonics Technol. Lett. 2003, 15, 1657-1659. [CrossRef]

26. Sun, X.; Chan, C.-K.; Wang, Z.; Lin, C.; Chen, L.-K. A single-fiber bi-directional WDM self-healing ring network with bi-directional OADM for metro-access applications. IEEE J. Sel. Areas Commun. 2007, 25, 18-24. [CrossRef]

27. Li, C.-Y.; Chang, C.-H.; Lin, Z.-G. Single-Line Bidirectional Optical Add/Drop Multiplexer for Ring Topology Optical Fiber Networks. Sensors 2021, 21, 2641. [CrossRef] [PubMed]

28. Keiser, G.E. A Review of WDM Technology and Applications. Opt. Fiber Technol. 1999, 5, 3-39. [CrossRef]

29. Jeruchim, M. Techniques for Estimating the Bit Error Rate in the Simulation of Digital Communication Systems. IEEE J. Sel. Areas Commun. 1984, 2, 153-170. [CrossRef] 